\title{
A Percepção da Equipe de Enfermagem na Avaliação da Dor em Unidades Pediátricas: Revisão Sistemática da Literatura
}

\author{
Eliane Moura da Silva ${ }^{1}$, Antônia Gomes de Olinda ${ }^{2}$, Francisco Hilângelo Vieira Barros ${ }^{3}$, \\ Leonardo Oliveira Silva ${ }^{4}$
}

\begin{abstract}
Resumo: A hospitalização é uma experiência traumática e estressante para qualquer indivíduo, especialmente para uma criança, e uso de um protocolo de assistência na Unidade Pediátrica (UP) propicia maior segurança aos usuários e profissionais. O objetivo do presente estudo é analisar se existe um protocolo que utiliza a metodologia do Processo Estratégico Situacional para auxiliar os enfermeiros na avaliação e manejo da dor em unidades pediátricas. Trata-se de um estudo de natureza qualitativa, descritiva por meia da Revisão Sistemática da Literatura (RSL) por meio de estudos publicados no período de 2008 a 2018. A seleção do estudo foi realizada a partir do descritor "Enfermagem Pediátrica" e encontrado 10564 resultados, sendo 594 artigos encontrados na base de dados SCIELO, 4342 artigos na base de dados LILACS, 3629 artigos encontrados na base de dados BDENF e 1999 artigos encontrados na base de dados MEDLINE. A análise permitiu a síntese dos dados, organizada em 03 categorias: Categoria A: cuidados essenciais para avaliação e manejo da dor sem a utilização de um protocolo. Categoria B: utilização de um protocolo para avaliação e manejo da dor a fim de diminuir possíveis traumas. Categoria C: O papel da enfermagem no cuidado sobre procedimentos invasivos em unidades pediátricas. O desenvolvimento do protocolo junto com a equipe de enfermagem facilita a avaliação e o manejo da dor, bem como tomada de decisões mais rápida e eficaz para o alívio da dor das crianças hospitalizadas nas UPs, sobretudo, proporcionar uma melhor qualidade da assistência de enfermagem.
\end{abstract}

Palavras-Chave: Dor. Enfermagem Pediátrica. Saúde da Criança.

\section{The Perception of the Nursing Team in the Evaluation of Pain in Pediatric Units: Systematic Review of the Literature}

\begin{abstract}
Hospitalization is a traumatic and stressful experience for any individual, especially a child, and use of a care protocol at the Pediatric Unit (UP) provides greater safety for users and professionals. The objective of the present study is to analyze if there is a protocol that uses the methodology of the Situational Strategic Process to assist the nurses in the evaluation and management of pain in pediatric units. This is a qualitative, descriptive study by means of the Systematic Review of Literature (RSL) by means of studies published in the period from 2008 to 2018.The study was selected from the descriptor "Pediatric Nursing" and found 10564 results, 594 articles found in the SCIELO database, 4342 articles in the LILACS database, 3629 articles found in the database BDENF and 1999 articles found in MEDLINE database. The analysis allowed the synthesis of the data, organized into 03 categories: Category A: essential care for assessment and management of pain without the use of a protocol.Category B: use of a protocol for evaluation and management of pain in order to reduce possible trauma. Category $\mathrm{C}$ : The role of nursing care in invasive procedures in pediatric units. The development of the protocol
\end{abstract}

\footnotetext{
${ }^{1}$ Mestre em Epidemiologia e Vigilância em Saúde pelo Instituto Evandro Chagas, Brasil. Enfermeira.

Contato: eliane82moura@ hotmail.com;

${ }^{2}$ Especialista em Enfermagem em UTI Pediátrica e Neonatal pela Faculdade Unyleya, Brasília-DF, enfermeira do Hospital Universitário da Grande Dourados-MS;

${ }^{3}$ Mestre em Saúde da Criança e do Adolescente pela Universidade Estadual do Ceará, Fortaleza-CE, enfermeiro do Hospital Universitário da Grande Dourados-MS. Contato: fhvbqxda@yahoo.com.br;

${ }^{4}$ Especialista em Enfermagem - Terapia Intensiva pela Faculdade do Trabalho, Uberlândia-MG, enfermeiro do Hospital Universitário da Grande Dourados-MS.
} 
together with the nursing team facilitates the assessment and management of pain, as well as faster and more efficient decision-making for the relief of the pain of hospitalized children in Ups, especially, to provide a better quality of nursing care.

Keywords: Pain. Pediatric Nursing. Child health.

\section{Introdução}

A hospitalização é uma experiência traumática e estressante para qualquer indivíduo, especialmente para uma criança (MARINEL; JARDIM, 2013). Com isso, a experiência dolorosa e a tensão a ela associada levam a danos físicos e emocionais que podem atrasar a recuperação e inclusive aumentar a mortalidade. Além do mais a avaliação da dor, sua profilaxia e seu tratamento são direitos básicos de todos, independentemente da idade do paciente (LEMOS; AMBIEL, 2010). Devido à complexidade dos cuidados as crianças internadas, devem-se lembrar da importância da adoção de medidas relacionadas com a avaliação e tratamento da dor (SILVA, 2011; ROCHA; PRADO, 2006).

O uso de um protocolo de assistência na Unidade Pediátrica (UP) propicia maior segurança aos usuários e profissionais, permitindo avaliar a dor como um sinal vital e oportuniza uma assistência mais humanizada. (MORAES, 2012).

Porém, estudos têm demonstrado que muitas vezes esse recurso não tem sido utilizado, ou ainda apenas por alguns profissionais esporadicamente, não sendo inseridos efetivamente na assistência aos pacientes (SANTANA, 2013).

Um dos objetivos fundamentais na admissão em uma UP é realizar o tratamento que minimize a ansiedade e a resposta à dor, causando a menor agressão possível na criança para evitar sofrimento físico e emocional. É evidente a importância da implementação de protocolos e estratégias em pediatria. O uso do protocolo promove a redução da variabilidade de ações de cuidado, melhorando na qualificação dos profissionais para a tomada de decisão assistencial. Ainda facilitam o desenvolvimento de indicadores de processo e de resultados (PIMENTA, 2015).

A utilização do protocolo pode auxiliar os enfermeiros na avaliação e manejo da dor o que tornaria mais fácil para reconhecer os sinais e sintomas da dor e através destes tratar as crianças com uma forma rápida e mais humanizada. 
Diante desse contexto, surgiu a seguinte questão norteadora: existe algum protocolo para avaliação e manejo da dor que a equipe de enfermagem utiliza nas UPs?

Através da Revisão Sistemática da Literatura (RSL) torna-se capaz de mostrar se existe de fato a aplicabilidade do protocolo nas UPs, uma vez que o uso desse instrumento poderá trazer melhorias na assistência de enfermagem com consequente melhora na evolução das crianças e redução dos traumas gerados pela hospitalização no paciente e na família. Portanto, o objetivo do presente estudo é analisar se existe um protocolo que utiliza a metodologia do PES para auxiliar os enfermeiros na avaliação e manejo da dor em unidades pediátricas.

\section{Revisão da Literatura}

\section{Definição de dor e sua fisiologia}

A dor é definida como uma experiência sensitiva e emocional desagradável, associada a um dano real ou potencial dos tecidos, ou descrita em termos de tais danos. (NASCIMENTO, 2011).

Nos últimos anos, importantes avanços ocorreram em relação à avaliação da dor com a validação de critérios objetivos, que hoje podem ser utilizados em diferentes locais e possibilitam as comparações de estudos. Foi de especial importância a padronização da dor como quinto sinal vital, pela Joint Commission on Accreditation of Healthcare Organizations (JCAHO); esta passou a considerar prioritárias a avaliação, intervenção e reavaliação da dor no processo de qualificação (acreditação) hospitalar (SAÇA; et al, 2010).

Entender cada processo da dor ajudará a reconhecer os fatores que causam a dor, os sintomas que acompanham a dor e a terapia selecionada (LEMOS; AMBIEL, 2010; SAÇA; et al, 2010).

Para avaliar a dor, o profissional de saúde é influenciado por aspectos relacionados à experiência profissional, facilidade de uso dos métodos de medição, tipo de dor, sinais de dor apresentados, idade da criança, tipo de procedimento doloroso, situação clínica, propriedades psicométricas, critérios de interpretação, facilidade de aplicação, experiência de utilização em outros serviços de saúde (MELO; et al, 2014; PETTENGILL, 2014).

A dificuldade na adoção de medidas de controle da dor está na falta de compreensão da comunicação não verbal e pelo fato de a dor ser um fenômeno subjetivo. Essas condições 
tornam a avaliação da dor na criança uma tarefa desafiante para os profissionais de saúde (MARTINS; et al, 2013).

O enfermeiro deve exercer seu papel no controle da dor, deve ser abordado de maneira sistemática para entender e tratar a dor do cliente. A capacidade para estabelecer um diagnóstico de enfermagem, decidir intervenções apropriadas e avaliar resposta do cliente às intervenções depende da atividade fundamental de uma avaliação factual, oportuna e rigorosa (SANTANA, 2013).

As duas escalas mais utilizadas em pediatria são as de Ramsay modificada (Quadro 1) e a de Comfort (Quadro 2) (SILVA, 2011).

Quadro 1: Escala de RAMSAY

\begin{tabular}{|l|}
\hline 1. Ansiedade e / ou agitação \\
\hline 2. Tranqüilidade, cooperação e orientação \\
\hline 3. Responsividade ao comando verbal \\
\hline 4. Resposta franca à estimulação auditiva ou compressão da glabela \\
\hline 5. Resposta débil à estimulação auditiva intensa ou compressão da glabela \\
\hline 6. Irresponsividade \\
\hline
\end{tabular}

Fonte: SILVA, M. S.; et al. Dor na Criança Internada: a percepção da equipe de enfermagem. Artigo Original. Rev. Dor. São Paulo, 2011.

Quadro 2: Escala de COMFORT

\begin{tabular}{|c|c|c|}
\hline Caracteristica & Avaliar & Pontos \\
\hline Estado de viglilia & $\begin{array}{l}\text { Muito sonolento } \\
\text { Luvemente sonolento } \\
\text { Acordado } \\
\text { Completamente acordado e alerta } \\
\text { Hiperalerta }\end{array}$ & $\begin{array}{l}1 \\
2 \\
3 \\
3 \\
5 \\
5\end{array}$ \\
\hline Agitaçăo & $\begin{array}{l}\text { Calmo } \\
\text { Levemente ansioso } \\
\text { Ansioso as } \\
\text { Muito ansioso } \\
\text { Pânico }\end{array}$ & $\begin{array}{l}1 \\
2 \\
3 \\
4 \\
4 \\
5\end{array}$ \\
\hline Resposta respiratória & 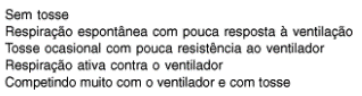 & $\begin{array}{l}1 \\
2 \\
3 \\
4 \\
5\end{array}$ \\
\hline Movimentos fisicos & $\begin{array}{l}\text { Sem movimentos } \\
\text { Leves movimentos ocasionais } \\
\text { Leves movimentos frequentes } \\
\text { Movimentos vitgorosos linitad os à extremidades } \\
\text { Movimentos vigorosos indusive do dorso e cabeça }\end{array}$ & $\begin{array}{l}1 \\
2 \\
3 \\
3 \\
5 \\
5\end{array}$ \\
\hline Pressão arterial (média) & $\begin{array}{l}\text { Abaixo do basal } \\
\text { Nomal } \\
\text { Aumentos raros de } 15 \% \text { do basal } \\
\text { Aumentros frequinetes de } 15 \% \text { do basal } \\
\text { Aumentos sustentados acima de } 15 \% \text { do basal }\end{array}$ & $\begin{array}{l}1 \\
2 \\
3 \\
3 \\
5 \\
5\end{array}$ \\
\hline Frequếncia cardiaca & $\begin{array}{l}\text { Abaixo do basal } \\
\text { Nomal } \\
\text { Aumentos raros de } 15 \% \text { do basal } \\
\text { Aumentos frequentes de } 15 \% \text { do basal } \\
\text { Aumentos sustentados acima de } 15 \% \text { do basal }\end{array}$ & $\begin{array}{l}1 \\
2 \\
3 \\
3 \\
4 \\
5\end{array}$ \\
\hline Tônus muscular & $\begin{array}{l}\text { Músculos totalmente relaxados } \\
\text { Tonnus muscular refuzzido } \\
\text { Tónus muscular nomal } \\
\text { Aumento do tônus muscular e flexão dos dedos } \\
\text { Rigidez muscular extrema e flexão dos dedos }\end{array}$ & $\begin{array}{l}1 \\
2 \\
3 \\
3 \\
5 \\
5\end{array}$ \\
\hline Tônus facial & 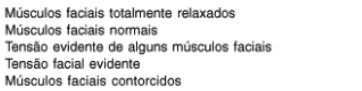 & $\begin{array}{l}1 \\
2 \\
3 \\
3 \\
5\end{array}$ \\
\hline
\end{tabular}

Sedaçăo excessiva 8-16; sedaçăo adequada 17-26; sedaçăo insuliciente 27-40.
Fonte: SILVA, M. S.; et al. Dor na Criança Internada: a percepção da equipe de enfermagem. Artigo Original. Rev. Dor. São Paulo, 2011 


\section{O Planejamento Estratégico Situacional}

O Planejamento Estratégico Situacional (PES) baseia-se no conhecimento prévio da realidade para elaboração de um plano em busca da modificação de um sistema, ele pressupõe respostas do sistema às ações para sua alteração, admite flexibilidade ao plano e não ignora as relações de poder e forças sociais que interferem em situações e problemas nos qual busca intervir (KLEBA; KRAUSER; VENDRUSCOLO, 2011).

O planejamento é um processo que envolve raciocínio e sensibilidade e requer conhecimentos de diversas complexidades a fim de intervir em problemas atuais e prever os futuros (DUTRA, 2013). Além do mais, engloba a tomada de decisão entre diferentes alternativas e poderá melhor subsidiar a tomada de decisão do enfermeiro. (PIMENTA, 2015).

\section{Sistematização da assistência de enfermagem (SAE) e o Processo de Enfermagem (PE)}

O PE é uma ferramenta metodológica utilizada para tornar a assistência de enfermagem sistemática, organizada em fases, com o objetivo de orientar o cuidado profissional de enfermagem, de promover a qualidade no cuidado prestado (NEVES; SHIMIZU, 2010).

Além do mais, o PE é considerado a base de sustentação da SAE, constituindo-se por fases ou etapas que envolvem a identificação de problemas de saúde do cliente, o delineamento do diagnóstico de enfermagem, a instituição de um plano de cuidados, a implementação das ações planejadas e a avaliação (ZANARDO; ZANARDO; KAEFER, 2011; BARRA; SASSO, 2010).

Dentre as diversas tecnologias presentes no ambiente das UPs, o Processo de Enfermagem (PE) destaca-se como uma tecnologia do cuidado que orienta a sequência do raciocínio lógico e melhora a qualidade do cuidado por meio da sistematização da avaliação clínica, dos diagnósticos, das intervenções e dos resultados de Enfermagem. O PE constitui-se numa ferramenta que deve ser utilizada pelos enfermeiros, pois evidencia o desencadeamento dos pensamentos e juízos desenvolvidos durante a realização dos cuidados, integra, organiza e garante a continuidade das informações da equipe de enfermagem permitindo avaliar a sua eficácia e efetividade e, modificá-la de acordo com os resultados na recuperação do cliente (SASSO; et al, 2012). 


\section{Protocolo de Enfermagem}

Protocolo é a descrição de uma situação específica de assistência/cuidado, que contém detalhes operacionais e especificações sobre o que se faz, quem faz e como se faz, conduzindo os profissionais nas decisões de assistência para a prevenção, recuperação ou reabilitação da saúde. Pode prever ações de avaliação/ diagnóstica ou de cuidado/tratamento, como o uso de intervenções educacionais, de tratamentos com meios físicos, de intervenções emocionais, sociais e farmacológicas, independentes de enfermagem ou compartilhadas com outros profissionais da equipe de saúde (PIMENTA, 2015; SANTOS; et al, 2013).

Há princípios estabelecidos para construção e validação de protocolos de assistência/cuidado, como a definição clara do foco, da população a que se destinam, quem é o executor das ações, qual a estratégia de revisão da literatura e análise das evidências utilizadas. Um protocolo deve descrever também a forma de validação pelos pares, estratégias de implementação e a construção dos desfechos ou resultados esperados (SANTANA, 2013).

Encontram-se na literatura diversos tipos de protocolos. Na área da saúde, podem ser citados diversos tipos como os relativos à assistência, aos cuidados, à organização do serviço, à atenção à saúde, ao acompanhamento e avaliação e também os protocolos clínicos entre outros. Essa diversidade de categorias pode ser agrupada, quanto à natureza, como protocolos clínicos e protocolos de organização dos serviços (PIMENTA, 2015).

Os protocolos clínicos têm foco na padronização de condutas clínicas, baseados em fundamentação técnica e científica, diretrizes organizacionais e políticas (SANTOS; et al, 2013). A instituição de protocolos clínicos integra a Sistematização da Assistência de Enfermagem (FELIX; SOARES; NÓBREGA, 2013).

Prática Baseada em Evidências (PBE) para construção de protocolos é o uso consciente, explícito e criterioso da melhor evidência disponível na tomada de decisões sobre o cuidado ao paciente. Esta prática requer a integração da melhor evidência disponível na literatura à experiência clínica do profissional, às preferências do paciente e aos recursos disponíveis na instituição. Além disso, fundamenta-se em pesquisas científicas. Avaliar a qualidade dos estudos requer dos profissionais conhecimentos sobre os diferentes tipos de estudos para a seleção dos melhores. Em relação aos tipos de estudo, tem-se, basicamente, quatro desenhos de pesquisa clínica: ensaio clínico, estudos de coorte, estudos caso-controle e transversal (BECK; POLIT, 2011). 


\section{Metodologia}

Trata-se de um estudo de natureza qualitativa e descritiva por meio de uma Revisão Sistemática da Literatura (RSL). O estudo foi iniciado com a formulação da pergunta norteadora, levando em consideração a problemática que envolve o tema, para conduzir o estudo. Este trabalho foi norteado pela seguinte questão: existe algum protocolo para avaliação e manejo da dor que a equipe de enfermagem utiliza nas UPs?

Após isso, houve a busca na literatura, que foi realizada na Biblioteca Virtual de Saúde, a partir dos Descritores em Ciências da Saúde (DeCS) da biblioteca virtual em saúde, utilizou na pesquisa o termo "Enfermagem Pediátrica" mais especificamente na Base de Dados da Literatura Latino-Americana e do caribe em Ciências da Saúde (LILACS), Scientific Eletronic Library Online (SCIELO), Base de Dados de Enfermagem (BDENF) e Medical Literature Analysis and Retrieval System Online (MEDLINE). A escolha das bases ocorreu devido à amplitude no que tange a abrangência dos periódicos.

Foram estabelecidos como critérios de inclusão utilizados para a seleção da amostra: estudos publicados no período de 2008 a 2018, texto completo na língua portuguesa, cujo país de publicação fosse o Brasil e o tema em questão envolvesse avaliação e manejo da dor em UPs; como critérios de exclusão: artigos que não disponibilizem acesso ao texto integral.

A coleta de dados ocorreu através da leitura ativa dos artigos para responder a objetivo. Além do mais, realizou-se a avaliação crítica dos artigos selecionados e verificado se respondiam plenamente a pergunta-guia. Avaliaram-se todas as produções encontradas, foram analisados o contexto histórico avaliação e manejo da dor em UPs pela equipe de enfermagem com utilização de protocolo. A observação crítica consistiu na fase onde todos os estudos selecionados foram avaliados com rigor metodológico (GALVÃO, 2004).

\section{Resultados e Discussão}

A seleção dos estudos foi realizada a partir do descritor "Enfermagem Pediátrica" e encontrados 10564 resultados, sendo 594 artigos encontrados na base de dados SCIELO, 4342 artigos na base de dados LILACS, 3629 artigos encontrados na base de dados BDENF e 1999 artigos encontrados na base de dados MEDLINE. Das publicações, 484 referências estavam relacionadas à avaliação e manejo da dor. Em seguida utilizaram-se os critérios de inclusão e exclusão, totalizando 58 artigos. Apenas 9 tratava-se sobre questão de utilização de protocolo. 
A análise permitiu a síntese dos dados, organizada em 03 categorias de acordo com o objetivo sendo: Categoria A: cuidados essenciais para avaliação e manejo da dor sem a utilização de um protocolo Categoria B: utilização de um protocolo para avaliação e manejo da dor a fim de diminuir possíveis traumas. Categoria C: O papel da enfermagem no cuidado sobre procedimentos invasivos em unidades pediátricas. Dessa maneira, possibilitou a obtenção de informações sobre o manejo e avaliação da dor e a discussão sobre as evidencias dos resultados.

A apresentação dos resultados é com base em cada um dos eixos e no quantitativo de referências.

\section{Categoria A: cuidados essenciais para avaliação e manejo da dor sem a utilização de um protocolo.}

Na categoria A, 29 produções $(59,18 \%)$ apontam sobre a quantidade de vezes que foi utilizado procedimentos invasivos no decorrer do tempo de internação nas UPs, que possivelmente geram traumas tanto para as crianças quanto para as famílias. As referências mostram o reconhecimento da magnitude do problema a nível global e citam o que foi feito no decorrer do tempo para amenizar tais problemas. Entre elas 7 referências $(58,33 \%)$ iniciam o contexto histórico citando sobre Charles Darwin em 1872 ao observar seus próprios filhos diante da dor (CHRISTOFFEL, 2012). Antigamente não se possuía uma assistência especializada e mesmo assim já analisavam os efeitos da dor, além de citar que em 1859, Florence Nightingale que demonstrava sua inquietação face a dor do paciente. (NASCIMENTO, 2015).

Além disso, 40 referências $(81,63 \%)$ mostram como funcionam o manejo da dor e quais os tipos de procedimentos invasivos são utilizados na UPs. Segundo Fidelis (2017) existe uma necessidade de avaliação, padronização do controle e sistematizar medidas para o alívio da dor farmacológicas e não farmacológicas. Percebeu-se que o propósito dessa iniciativa foi determinar e identificar prioridades nas UPs. Em se tratando do manejo da dor, bem como contribuir para a pesquisa no campo, ou seja, nesse contex to as instituições hospitalares estavam cada vez mais preocupadas em garantir um atendimento de qualidade a seus clientes implementando medidas para diminuir traumas e para compreensão da dor na criança de acordo com as especificidades de cada uma. 
Na pesquisa 10 referências $(20,4 \%)$ procedimentos que poderiam ser programados ou esperados como Cateter Central de Inserção Periférica (PICC), por exemplo, bem como preservar o membro para o PICC aumentando as chances de acerto. Além disso, 36 publicações (73, 46\%) abrangiam sobre a utilização de Solução Oral de Glicose (SOG) para coleta de sangue, PICC ou outros tipos de punções. A SOG vem sendo utilizada no tratamento da dor com resultados favoráveis durante procedimentos que geram dor de intensidade leve a moderada ou como adjuvante no tratamento da dor intensa (MARCATTO, 2011).

Nos últimos tempos, muitas pesquisas ocorreram na abordagem da dor, mas, procedimentos que causam dor leve a moderada ainda tendem a ser pouco valorizados na rotina das unidades e consequentemente, subtratados. A glicose é um dos recursos mais indicados nestas situações. Os procedimentos nos quais a SOG tem sido utilizada são: punção venosa e arterial, punção do calcanhar, punção lombar, instalação de cateter percutâneo, dissecções venosas, injeções subcutâneas e intramusculares, remoção de adesivos e fitas da pele e remoção de drenos (MORAES, 2012). Também 32 referências $(65,3 \%)$ trata-se sobre o uso de sucção não nutritiva, pois quando associada à sucção SOG, ocorre um sinergismo que pode resultar em um controle mais efetivo dos sinais indiretos de dor (COSTA, 2016).

Todavia 28 artigos $(57,14 \%)$ referiam sobre promover posicionamento adequado através de rolos confortáveis que ofereçam limites e alinhamento corporal com equilíbrio entre posturas flexoras e extensoras, entre outros de acordo com o procedimento a ser realizado. Segundo Moura e Gonçalves (2010) essa organização de manejos, sejam ou não sejam farmacológicos, são feitos antes de iniciar um procedimento, fazendo com que o paciente não sinta a dor que sentiria. Esses recursos auxiliam o combate ao estimulo nociceptivo, que são os estímulos dolorosos que ele recebe.

\section{Categoria B: utilização de um protocolo para avaliação e manejo da dor a fim de diminuir possíveis traumas.}

Nesta categoria 7 artigos $(77,8 \%)$ discorrem sobre o tema de acordo com os fatores que influenciam na avaliação e manejo da dor em UPs, onde verificou-se que os artigos, em algum momento, tratam da padronização de protocolo em UPs, 50\% das referências abordam que existe a monitorização do parâmetro da dor uma vez por turno, ou seja, a cada 6 horas, no entanto ainda não há o uso de um protocolo propriamente dito. Além do mais, 5 artigos 
$(55,56 \%)$ tratam do assunto que o protocolo está em fase de construção para s melhorar o manejo da dor em UPs. Também cita que com o uso do protocolo pode reduzir o risco de possíveis traumas causados por procedimentos invasivos.

Sobretudo, 8 produções $(88,89 \%)$ enfatizam que o uso do protocolo proporciona uma melhor qualidade e eficácia na assistência das UPs. No entanto, a utilização do protocolo é multiprofissional, não tendo uma ferramenta exclusiva para os enfermeiros sobre a avaliação da dor. Todas as referências mencionam que em algum momento os enfermeiros utilizam escalas como a de CONFORT e a de RAMSAY para avaliar a dor.

Portanto para garantir uma assistência de qualidade, o profissional da área da saúde deve ter uma visão holística do cuidado, tendo a avaliação e manejo da dor como foco e contemplando aspectos referentes não somente ao cuidar, mas também ao educar e pesquisar (SILVA, 2011). Leva-se em consideração que a educação, o treinamento dos profissionais e a realização de pesquisas sobre o tema favorecerão a divulgação para padronização de um protocolo em, com vista a reduzir ao máximo os eventos adversos e, consequentemente, as práticas inseguras que colocam em risco a saúde de pacientes e profissionais. Ou seja, ao se falar em avaliação e manejo da dor reafirma-se a necessidade de mudança de comportamento por parte dos profissionais como um aspecto essencial.

\section{Categoria C: O papel da enfermagem no cuidado sobre procedimentos invasivos em unidades pediátricas.}

Barra (2010) constatou que, apesar de poucos enfermeiros usarem instrumentos padronizados (escalas) para avaliar a intensidade da dor, as taxas da prevalência da dor comparadas com as taxas de tratamento parecem comprovar que os enfermeiros reconhecem manifestações de dor no recém-nascido e tomam medidas para o seu controlo. No decorrer da pesquisa verificou-se que em 37 referências $(63,79 \%)$ afirmam que os enfermeiros reconhecem a criança com dor e atuam implementando intervenções de enfermagem que visam o controlo da dor. Só depois efetua a respetiva avaliação da dor com recurso à escala de dor.

Neste contexto a enfermagem desempenha um papel fundamental, tanto pelo seu contingente como pela sua proximidade constante e ininterrupta na assistência ao paciente, estando apta a identificar quando a criança está sentindo algum desconforto, bem como a oferecer valiosas sugestões de melhoria. Sendo assim, essa proximidade pode acabar 
levantando questionamentos sobre a condição da criança e sobre as melhores formas de publicação de trabalhos científicos.

A presente pesquisa demonstrou que 28 referências $(88,88 \%)$ mencionam que a equipe de Enfermagem é fundamental para a identificação dos diversos riscos aos quais o paciente está exposto e a partir disso adotam condutas padronizadas com o intuito de prevenir o dano, tanto na atenção básica quanto hospitalar.

Em outra perspectiva, 17 referências $(29,31 \%)$ abordam a prática baseada em evidência como ferramenta para a atuação do enfermeiro. Constatou-se que a implementação da evidência clínica na prática é tarefa difícil, sugerindo treinamentos a serem desenvolvida para utilização do protocolo a fim de atingir o êxito na avaliação e manejo da dor. Além de desenvolver competências para interpretar os resultados das pesquisas e capacitação periódica da equipe.

Com base nas reflexões, ressalta-se que a qualidade da assistência ao paciente resulta em uma crescente participação da equipe de enfermagem.

\section{Conclusão}

Conclui-se que a hospitalização é um momento estressante para uma criança que pode levar danos físicos e emocionais podendo atrasar na recuperação da mesma; com isso aumentando o índice de mortalidade.

Portanto devem-se adotar medidas para avaliação e tratamento da dor usando meios de cuidados a saúde, e as tecnologias disponíveis promovendo um acolhimento e respeito ético pelas crianças internadas nas UPs. O uso de protocolos proporciona maior segurança aos profissionais para avaliar a dor como um sinal vital podendo assim oferecer uma assistência mais humanizada.

Dado o exposto, $\mathrm{O}$ desenvolvimento do protocolo junto com a equipe de enfermagem facilita a avaliação e o manejo da dor, bem como tomada de decisões mais rápida e eficaz para o alívio da dor das crianças hospitalizadas nas UPs. Sobretudo, proporcionar uma melhor qualidade da assistência de enfermagem. Por todos esses aspectos, esta pesquisa também poderá assegurar subsídios para a realização de novas pesquisas relacionadas à utilização de protocolos visando avaliação e melhoria continua da assistência prestada. 


\section{Referências}

BARRA, D. C. C.; SASSO, G. T. M. D. Tecnologia Móvel à Beira do Leito: processo de enfermagem informatizado em terapia intensiva a partir da CIPE. Texto Contexto Enferm, Florianópolis, 2010. Acesso em: 26 jul. 18. Disponível em: http://www.scielo.br/pdf/tce/v19n1/v19n1a06.

BECK, C.T.; POLIT, D.F. Fundamentos de Pesquisa em Enfermagem: avaliação de evidências para prática de enfermagem. Ed. Artmed $7^{\circ} \mathrm{ed}, 2011$.

COSTA, L.C. et. al. Utilização de Medidas Não Farmacológicas pela Equipe de Enfermagem para Alívio Da Dor em Unidades Neonatal. Rev enferm UFPE on line., Recife, 10(7):2395-403, jul., 2016.

DUTRA, H.S. O Planejamento e sua aplicação em Enfermagem. Juiz de Fora- MG, 2013. D isponível em: < http://www.ufjf.br/admenf/files/2013/09/Aula-6-O-Planejamento-e-sua-aplica\%C3\%A7\%C3\% A3o-em-Enfermagem-11-nov-2013.pdf $>$ Acesso em: 26 jul. 18.

FIDELIS, W.M. Z.; KANAI, K. Y. Conhecimento e Percepção da Equipe de Enfermagem em Relação à Dor na Criança Internada. Rev Dor ;11(1):20-27. São Paulo/SP, 2010. Disponível em: < http://files.bvs.br/upload/S/1806-0013/2010/v11n1/a1495.pdf> Acesso em: 15 mai .2018.

KLEBA, M. E.; KRAUSER, I. M.; VENDRUSCOLO, C. O Planejamento Estratégico Situacional no Ensino da Gestão em Saúde da Família. Texto Contexto Enferm, Sistema de Información Científica Red de Revistas Científicas de América Latina y el Caribe, España y Portugal. Florianópolis, 2011 Jan-Mar; 20(1): 184-93

LEMOS, S.; AMBIEL, C. R. Dor em Pediatria: fisiopatologia, avaliação e tratamento. Rev. Saúde e Pesquisa, v. 3, n. 3, p. 371-378, set. /Dez. 2010.

LUIZ, F.F. A sistematização da Assistência de Enfermagem na Perspectiva da Equipe de um Hospital de Ensino. Rev. Eletr. Enf. [Internet]. 2010; Disponível em:

< file:///C:/Users/eliane/Downloads/8642-51335-1-PB.pdf > Acesso em: 27 jul. 18

MARCATTO, J.O. et. al. Benefícios e limitações da utilização da glicose no tratamento da dor em neonatos: revisão da literatura. Rev. bras. ter. intensiva vol.23 no.2 São Paulo April/June 2011.

MARINELO, G. S.; JARDIM, D. P. Estratégias lúdicas na assistência ao paciente pediátrico: aplicabilidade ao ambiente cirúrgico. Rev. SOBECC, São Paulo. abr./jun. 2013.

MARTINS, S.W.; et al. Avaliação e controle da dor por enfermeiras de uma unidade de terapia intensiva neonatal. Rev. dor vol.14 no.1 São Paulo Jan./Mar. 2013. Disponível em:http://www.scielo.br/scielo.php?pid=S180600132013000100006\&script=sci_arttext\&tlng=pt> Acesso em: 18 jul. 2018.

MELO, G. M.S.; et al. Escalas de avaliação de dor em recém-nascidos: revisão integrativa. Rev. Paul. Pediatr, 2014.

MORAES, L. V. L. Proposta de Protocolo de Interrupção Diária da Infusão Contínua de Sedativos em Unidade de Terapia Intensiva Pediátrica. Brasília- DF, 2012.

NASCIMENTO, L. A.; et al. Manejo da Dor e Dificuldades Relatadas Pela Equipe de Enfermagem na Administração de Opióides. Rev. Eletr. Enf. [Internet]. 2011 out/dez;13(4):714-20. 
NEVES R. S.; SHIMIZU, H. E. Análise da implementação da Sistematização da Assistência de Enfermagem em uma unidade de reabilitação Enfermagem em uma unidade de reabilitação. Rev Bras Enferm, Brasília, 2010 mar-abr; 63(2): 222-9

PIMENTA, C. A. M.; et al. Guia para Construção de Protocolos Assistenciais de Enfermagem. COREN- São Paulo- SP, 2015.

ROCHA, P.K.; PRADO, M. L.; SEBOLD, L. F. Programas de Atualização em Enfermagem Saúde da Criança e do Adolescente-PROENF. Editora Artamed. Porto Alegre/ RS, 2006.

SAÇA, C.S.; et al. A Dor como $5^{\circ}$ Sinal Vital: atuação da equipe de enfermagem no hospital privado com gestão do Sistema Único de Saúde (SUS). Health Sci Inst. São Paulo- SP, 2010. Acesso em: 15 jun. 18 Disponível em: < http://200.196.224.129/comunicacao/publicacoes/ics/edicoes/2010/01_janmar/V28_n1_2010_p35-41.pdf>

SANTANA, R.F. Seleção de Medicamentos: indicadores, estratégias de implantação e contribuições para o Sistema Único de Saúde. São Cristóvão/SE, 2013.Disponível em:<https://bdtd.ufs.br/bitstream/tede/1057/1/RAFAEL_SANTOS_SANTANA.pdf\#page=44 Acesso em: 25 jul.2018

SANTOS, C.T.; et al. Indicador de Qualidade Assistencial Úlcera por Pressão: análise de prontuário e de notificação de incidente. Rev. Gaúcha de Enfem. 2013.Acesso em: 20 jun. 18 Disponível em: < https://www.lume.ufrgs.br/bitstream/handle/10183/85388 /000879360.pdf?sequence=1>

SASSO, G.T.M.D;.et al. Processo de Enfermagem Informatizado: metodologia para associação da avaliação clínica, diagnósticos, intervenções e resultados. Rev Esc Enferm USP, 2012

SILVA, M. S.; et al. Dor na Criança Internada: a percepção da equipe de enfermagem. Artigo Original. Rev. Dor. São Paulo, 2011.

ZANARDO, G. M.; ZANARDO, G.M.; KAEFER, C. T. Sistematização da Assistência de Enfermagem. Rev. Contexto e Saúde. Ed. Unijui. Vol $10 \mathrm{n}^{\circ}$ 20. 2011. Disponível em: < file:///C:/Users/eliane/Downloads/1811-7417-1-PB.pdf > Acesso em: 05 jul. 18

\section{Como citar este artigo (Formato ABNT):}

SILVA, Eliane Moura da; OLINDA, Antônia Gomes de; BARROS, Francisco Hilângelo Vieira; SILVA, Leonardo Oliveira. Percepção da Equipe de Enfermagem na Avaliação da Dor em Unidades Pediátricas: Revisão Sistemática da Literatura. Id on Line Rev.Mult. Psic., 2018, vol.12, n.42, Supl. 1, p. 777-789. ISSN: 1981-1179.

Recebido: 14/11/2018;

Aceito: 17/11/2018 